\title{
Análise e visualização de dados de rastreamento para caracterização da logística urbana
}

\section{Patrícia Faias Laranjeiro de Andrade ${ }^{1}$, Leonardo Alves Godoy ${ }^{2}$, Mariana Abrantes Giannotti ${ }^{3}$, Claudio Barbieri da Cunha ${ }^{4}$, Hugo Tsugunobu Yoshida Yoshizaki ${ }^{5}$}

\author{
${ }^{1}$ Universidade de São Paulo, patricia.laranjeiro@usp.br \\ 2Universidade de São Paulo, leonardo.godoy@usp.br \\ 3Universidade de São Paulo, mariana.giannotti@usp.br \\ 4Universidade de São Paulo, cbcunha@usp.br \\ 5Universidade de São Paulo, hugo@usp.br
}

\section{Recebido:}

31 de março de 2017

Aceito para publicação:

18 de agosto de 2017

Publicado:

29 de outubro de 2017

Editor de área:

Bruno Vieira Bertoncini

\section{Palavras-chaves:}

Logística urbana,

Big data geoespacial,

Dados GPS,

Visualização de dados.

\section{Keywords:}

Urban logistics,

Geospatial big data,

GPS data,

Data visualization.

DOI:10.14295/transportes.v25i3.1353

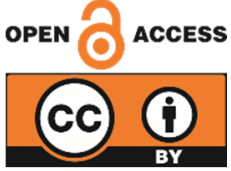

\section{RESUMO}

Este estudo tem como objetivo aplicar técnicas de análise e visualização de dados GPS (Global Positioning System) para entender o comportamento do transporte urbano de carga em algumas capitais do país, através de indicadores logísticos como distribuição dos pontos de entrega, distribuição do volume de caminhões ao longo do tempo (dia da semana e hora do dia), etc. Para tanto, utilizaram-se duas bases de dados de rastreamento de veículos, distintas, sendo a primeira fornecida por duas empresas varejistas que já possuíam tais dados por questões de segurança e monitoramento do comportamento dos motoristas, e a segunda disponibilizada por uma empresa que presta serviços de fornecimento de mapas e possui dados de diversas empresas, em sua maioria prestadores de serviço de monitoramento de veículos. Para o processamento dos dados foram usadas ferramentas de análise de grandes volumes de dados geoespaciais (geospatial big data). Para ambas as bases foi possível analisar, para o período de 06 a 10 de outubro de 2014 (segunda à sexta), o padrão de distribuição dos caminhões por dia da semana, cujas análises apontam para uma quantidade menor de veículos circulando na segunda-feira, em relação aos outros dias da semana (terça à sexta).

\section{RESUMO}

This study aims to apply GPS (Global Positioning System) data analysis and visualization techniques in order to understand the urban logistics' behavior in some cities of Brazil, through logistical indicators such as distribution of delivery points, volume of distribution of trucks over time (day and time of day), etc. For that, two distinct databases of vehicle tracking were used: the first provided by two large retailers that already had such data for risk management and monitoring of drivers' behavior; and the second one belongs to a map services provider whose data is derived from several companies, mostly vehicle tracking companies. To process this amount of data, it was necessary to use geospatial big data analysis tools. In both databases, it was possible to analyze, for the period 06-10 of October 2014 (Monday to Friday), the volume distribution pattern per day, and the results point to a smaller volume of vehicles circulating on Monday in comparison to other working days (Tuesday to Friday).

\section{INTRODUÇÃO}

Os congestionamentos de trânsito são um dos grandes problemas que afetam as grandes cidades. Tais aglomerações urbanas ganham considerável atenção devido ao seu tamanho populacional e suas influências econômica, sociocultural, ambiental e política.

Em tais centros urbanos vem-se observando crescente consumo e movimentação de produtos, tornando imperativo buscar soluções para uma utilização mais eficiente da escassa infraestrutura viária urbana. É importante destacar ainda, a tendência de que o tráfego urbano de veículos de carga venha a 
aumentar ainda mais, motivado não só pelo crescimento econômico, mas também por outros fenômenos contemporâneos recentes como o comércio eletrônico, que permite que as pessoas comprem e recebam produtos sem sair de casa. Isso representa um desafio para as cidades, que tem como objetivo compatibilizar a necessidade de abastecimento com os fluxos de pessoas que necessitam se deslocar por suas vias.

Assim, os impactos negativos do transporte de carga, como trânsito congestionado e altas taxas de poluição, são comuns nas cidades modernas e impulsionam medidas e políticas direcionadas para mitigá-los. Uma das medidas mais comumente adotadas, não só em cidades brasileiras, mas também em outras partes do mundo, é a restrição de circulação de veículos de carga, que proíbe os mesmos de circular (usualmente em determinados horários e dias específicos) e, às vezes, até de realizar operações de carga e descarga, principalmente em áreas mais centrais das grandes cidades (Bontempo et al., 2014). Entretanto, nota-se que tal medida, assim como a maioria das medidas e políticas relacionadas à logística urbana, são tomadas sem um estudo mais aprofundado dos seus impactos antes de sua implementação, e muitas vezes os impactos não são mensurados e acompanhados a posteriori, o que torna questionável a sua eficácia e eficiência e dificulta a sua manutenção a longo prazo.

Neste contexto, é imprescindível entender o comportamento do transporte de carga urbano com o intuito de otimizá-lo e subsidiar a formulação de novas políticas públicas que tragam benefícios efetivos tanto ao setor privado como à sociedade como um todo. Para tanto, são necessários mais dados e informações para que se possa desenvolver modelos de transporte representativos.

Há diversas formas de se obterem tais informações, porém as mais tradicionais envolvem levantamentos de campo, como a pesquisa origem-destino de cargas, que são custosas e demoradas. Houve, nas últimas duas décadas, uma crescente popularização de dispositivos GPS, tornando frequente o seu uso para o rastreamento dos veículos de carga, motivado principalmente por necessidades de gerenciamento de segurança; tal fato permitiu o início do uso desses dados para extrair informações operacionais do transporte de carga. A utilização desses dados de rastreamento, no entanto, requerem um conjunto de técnicas e metodologias para lidar com os grandes volumes de dados com características geoespaciais (conhecidos como geospatial big data). Métodos relacionados à exploração de grandes volumes de dados, em especial os espaciais, ainda não estão consolidados na comunidade científica e, nesse sentido, constituem um profícuo espaço para investigações científicas.

Neste trabalho, a partir de duas bases distintas de dados de rastreamento de veículos referentes a uma semana de outubro de 2014, são apresentadas análises desenvolvidas com o objetivo de um melhor entendimento sobre a distribuição urbana na cidade de São Paulo, que também inclui uma comparação com outras capitais do Brasil. Entre outras questões, os dados são explorados a fim de se determinar, por exemplo, qual a distribuição espacial dos veículos nos diferentes dias e horários ao longo da semana; em outras palavras, onde se concentram e por onde se distribuem no tempo e no espaço os caminhões na cidade de São Paulo, ou se existem diferenças na quantidade de veículos de carga circulando nos diferentes dias da semana. Em que horários esses veículos operam? Existem diferenças entre as capitais e quais são elas?

Cabe ressaltar o ineditismo deste estudo com foco na utilização de dados de rastreamento voltada ao entendimento da logística urbana e comparação de várias cidades, com a finalidade de coletar elementos para um diagnóstico que possa, inclusive, embasar políticas públicas.

\section{REVISÃO BIBLIOGRÁFICA}

A revisão bibliográfica deste trabalho está dividida em duas partes: a primeira referente à análise de dados de rastreamento (GPS) e a segunda referente à visualização de dados, que tem sido apontada como preponderante para a análise de grandes volumes de dados espaciais, como será discutido nesta seção. 


\subsection{Análise de dados GPS}

Com o avanço da tecnologia e a difusão do uso de dispositivos de rastreamento de veículos, principalmente para gerenciamento de risco e avaliação do comportamento do motorista (Greaves e Figliozzi, 2008), surgiu a possibilidade de aproveitar dados obtidos através de sistemas de navegação por satélite (Sistema Global de Navegação por Satélite, em inglês GNSS - Global Navigation Satellite System), sendo o mais comum o GPS; para extrair informações sobre o comportamento do transporte de carga.

A obtenção de dados de GPS para o estudo e planejamento do transporte de carga urbano apresenta algumas vantagens sobre os métodos de obtenção de dados tradicionais, como: a maior precisão em relação aos dados obtidos de pesquisas, menor ou nenhuma dependência de interação com o motorista; o baixo custo de aquisição destes dados bem como a facilidade de obtê-los para um longo período de tempo (Joubert e Meintjes, 2015).

Entretanto, pode-se citar também fatores externos que podem prejudicar a coleta de dados GPS, como a existência de muitos edifícios no meio urbano, que interferem na quantidade de satélites visíveis, podendo afetar sua precisão ao fornecer o posicionamento (latitude, longitude, altitude) que depende do número de satélites disponíveis, sendo necessário um mínimo de quatro satélites para determinar a localização (Joubert e Meintjes, 2015).

Outra dificuldade ao se utilizar dados GPS consiste no fato de ser necessário um pré-processamento para eventuais correções de erros nos dados brutos coletados pelos aparelhos GPS, além de uma correta padronização para a construção de uma base de dados consolidada com uma estrutura que permita o estudo das trajetórias individuais, coletivas ou mesmo de forma sumarizada de diversos objetos no tempo. Isso somente se torna possível com o correto registro das trajetórias de um objeto, em outras palavras, uma sequência espaço-temporal ordenada em um dado intervalo de tempo finito (Spaccapietra et al., 2013). Somente então é possível extrair informações relevantes a partir dos dados brutos, como tempos de deslocamento e tempos de parada, identificação dos pontos e tempos de entrega, velocidades calculadas a partir do deslocamento e do intervalo de tempo, etc. Porém, devido à enorme quantidade de dados nas bases, o pré-processamento e o processo de mineração de dados de objetos em movimento - mobility data mining - (Nanni, 2013) são dispendiosos, requerendo recursos de hardware de alta performance (high performance computing) e elevada capacidade de armazenamento, assim como estratégias, utilização e desenvolvimento de aplicações em plataformas específicas para a análise de bases com grande volume de dados.

É de grande utilidade, neste caso, a utilização de ferramentas de big data, que pressupõem o tratamento de dados com grande volume, velocidade e variedade (Lee e Kang, 2015). 0 fato das bases utilizadas neste trabalho serem estáticas, ou seja, não possuem fluxo contínuo de entrada de registros, e nem haver grande variedade em seus tipos de dados e origens não torna menos massivo e desafiador todo o processo a ser executado.

\subsection{Visualização de dados}

0 processo de tornar dados analíticos em dados visuais é essencial, no sentido de tornar possível a compreensão e interpretação destes dados por agentes humanos. É, portanto, importante que sejam escolhidos processos adequados e modelos eficientes para garantir a ilustração clara da informação.

Segundo o modelo proposto por Chi (2000), os dados podem ser categorizados em um fluxo de quatro estágios: dados brutos, abstrações analíticas, abstrações visuais e imagem. Para que esses dados sejam transformados de um estágio para o outro, utiliza-se operadores específicos (intra e inter-estágios), devendo ser combinados de acordo com as intenções da modelagem. Logo, para determinar-se a forma de visualização mais conveniente é importante identificar a forma e o conteúdo inicial dos dados utilizados, também como o tratamento a que serão submetidos.

No que se trata de dados coletados através de dispositivos de rastreamento, estes são de natureza 
estática e apresentam componentes independentes quais sejam, o geoespacial, o temporal e o identificador, que podem estar associadas outras variáveis dependentes correspondentes. Por exemplo, sendo um dado associado a um veículo, seu componente identificador poderá fornecer o tipo de veículo, ano de fabricação, número de revisões realizada, entre outras informações, desde que associada corretamente a esse segundo conjunto.

A característica geoespacial do dado implica em determinações relativas ao modelo e ferramentas utilizados para sua visualização. Usualmente trabalha-se em um domínio de referência, relativo a um atributo; no contexto geo-visual este conceito deve ser estendido, apresentando um segundo domínio de referência: o geográfico, impondo um conjunto de dois focos (Schumann e Tominski, 2011). Além disso, dados geoespaciais sempre foram parte do big data (Lee e Kang, 2015), existindo na atualidade ferramentas cada vez mais capazes de lidar com dados em grande volume, velocidade e variedade.

Dadas as especificidades das informações tratadas, o modelo de transformação de dados analíticos em visuais (mapeamento) e destes em imagens (renderização) (Chi, 2000) deverá apresentar condições compatíveis. Mapas coropléticos (Kraak e Ormeling, 1996), cartogramas (Dorling et al., 2006) e representações em três dimensões (3D) (Tominski et al., 2005) são considerados meios clássicos de visualização de dados de múltiplas variáveis. Por causa do imenso tamanho das atuais bases de dados, muitas técnicas voltam-se a visualização de abstrações (Schumann e Tominski, 2011). Estudos recentes também têm abordado a questão de como encontrar formas adequadas especificamente para a geovisualização de conjuntos complexos de dados e modelos (Loidl et al., 2016).

Busca-se que a visualização dos dados apresente visibilidade, nível e qualidade de conteúdo adequados. Uma visualização de formato adequado deve proporcionar melhores análises, observações e consequentes inferências e previsões, especialmente no setor de modelos. Observa-se um potencial subutilizado para usos da geovisualização na modelagem de sistemas de transporte (Loidl et al., 2016).

\section{DESCRIÇÃO DOS DADOS}

Com o objetivo de buscar diagnosticar e avaliar alguns aspectos da logística urbana em São Paulo, e também em outras grandes capitais brasileiras, foram utilizados, principalmente, dados de rastreamento de veículos, captados através de dispositivos GPS. Trata-se de uma proposta nova, uma vez que não foram encontrados trabalhos similares com essa finalidade na literatura sobre o assunto. Neste âmbito, foram utilizadas duas bases distintas, ambas fornecidas exclusivamente para desenvolvimento de pesquisas acadêmicas.

A primeira base de dados (denominada Base A) consiste nos dados de rastreamento de todos os veículos de carga de duas empresas distintas, ambas grandes redes varejistas, para todo o Brasil, referente ao mês de outubro de 2014. Para esta base, sabe-se que todos os veículos utilizados para o transporte destas empresas são caminhões, porém não há nenhuma informação sobre os tipos e modelos específicos de caminhões.

A segunda base de dados (denominada Base B) constitui uma base de rastreamento de diversos tipos de veículos (caminhões, táxis, ônibus, automóveis) também para todo o país, referente ao período de 06 a 10 de outubro de 2014 (segunda à sexta), disponibilizada por uma empresa que fornece mapas e serviços correlatos. Nesse caso, por uma questão de confidencialidade, os dados disponibilizados para a pesquisa não possuem a identificação das empresas que pertencem tais dados, nem seu ramo de atividade, ou quais veículos pertencem a cada empresa.

Apesar de serem bases de dados diferentes pelos aspectos citados acima, ambas apresentam as informações consideradas essenciais para desenvolver-se uma análise espaço-temporal, sendo esses:

- Posição geográfica: latitude e longitude do registro;

- Data e hora do registro;

- Código único ("chave") de identificação de cada veículo (Denominado "ID veículo") 


\section{ABORDAGEM METODOLÓGICA}

A abordagem utilizada neste trabalho englobou as seguintes etapas, cuja sequência lógica é mostrada na Figura 1:

- Identificação de indicadores do perfil de transporte urbano de carga;

- Identificação de técnicas de processamento e visualização de dados GPS;

- Coleta de dados de GPS: obtenção de dados de rastreamento de veículos de empresas;

- Aplicação das técnicas de processamento e visualização de dados GPS;

- Cálculo dos indicadores do perfil do transporte de carga;

- Análise dos resultados.

Cabe ressaltar que a primeira etapa do processamento dos dados corresponde ao chamado "Data Cleaning", que consiste na limpeza e uniformização dos dados, basicamente através da eliminação de outliers, ou seja, registros atípicos que estão muito distantes das demais observações. A definição dos outliers baseou-se na literatura (que é limitada) e também na expertise da equipe, sendo estes os critérios adotados e suas respectivas justificativas:

- Fase 1:

- Dados sem valor em um ou mais campos (latitude/longitude, data/hora, ID veículo): impossibilitam os cálculos de um ou mais parâmetros necessários para as análises espaçotemporais;

- Dados duplicados: registros com todos os campos com valores idênticos (latitude/longitude, data/hora, ID veículo): são registros advindos de algum erro do aparelho de rastreamento.

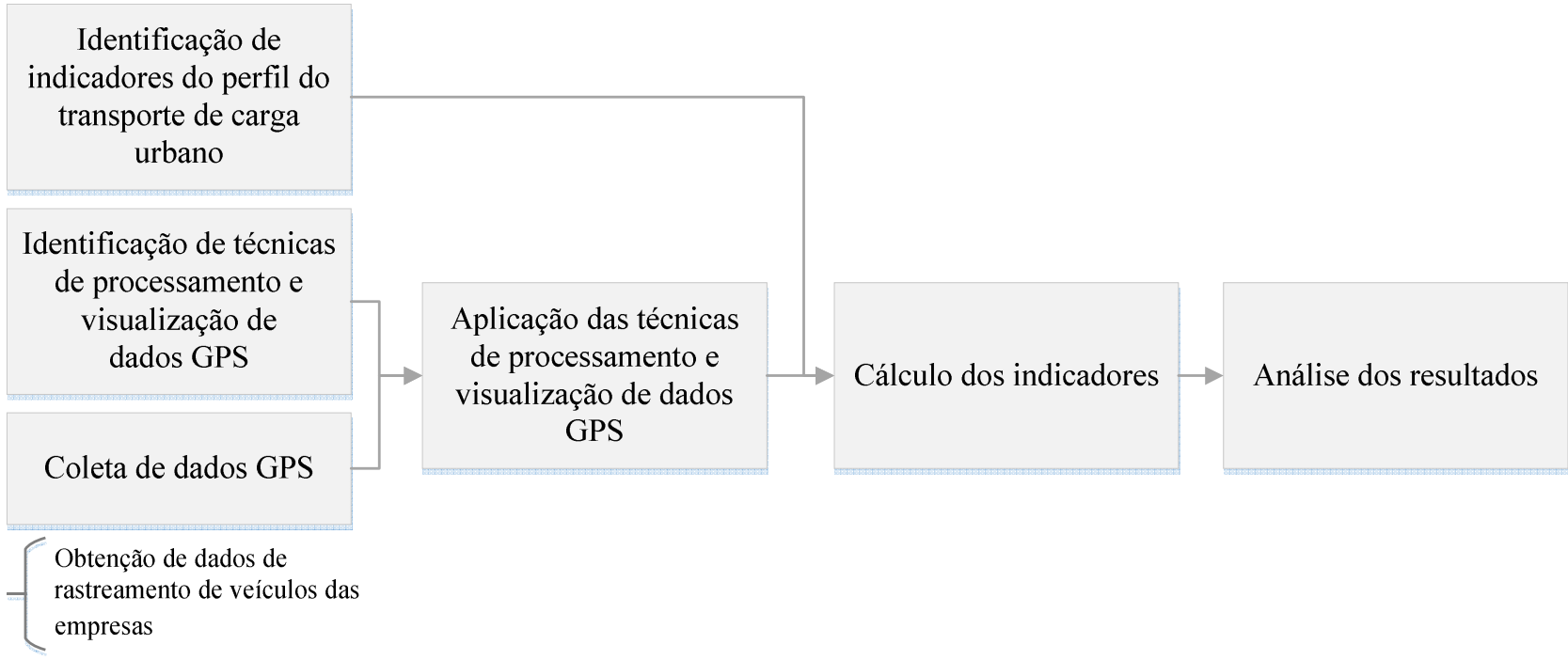

Figura 1: Sequência Lógica da Abordagem Metodológica para a Análise dos Dados de GPS

- Fase 2:

- Registros com velocidades superiores a $110 \mathrm{~km} / \mathrm{h}$ : registros com tais velocidades mostram um possível erro do aparelho de rastreamento, dado que as velocidades máximas permitidas nas vias não ultrapassam $90 \mathrm{~km} / \mathrm{h}$. Adotou-se $110 \mathrm{~km} / \mathrm{h}$ como a velocidade de corte a partir da análise do histograma de velocidades dos veículos e considerando a possibilidade de alguns veículos circularem a uma velocidade um pouco acima da permitida; 
- Registros cujo módulo da aceleração seja superior a $10 \mathrm{~m} / \mathrm{s}^{2}$ : tal aceleração implica que o veículo aumentasse ou diminuísse a sua velocidade em $36 \mathrm{~km} / \mathrm{h}$ em 1 segundo, o que não é fisicamente possível.

- Veículos com menos de 5 registros ao todo no período analisado (5 dias - 06 a 10 de outubro de 2014): nesses casos todos os registros do veículo foram descartados, pois é uma quantidade pouco significativa.

Foram considerados os registros dos veículos de carga circulando dentro do município de São Paulo, Rio de Janeiro, Belo Horizonte, Curitiba, Recife e Salvador. O procedimento de "Data Cleaning" descrito acima resultou no descarte de cerca de $5 \%$ do total de registros.

Para fins de comparação dos padrões de deslocamento dos caminhões, conjuntamente para as Bases A e B, utilizou-se o período coincidente entre essas, correspondente a uma semana (segunda à sexta): 06 a 10 de outubro de 2014 .

\section{RESULTADOS}

Os resultados obtidos serão apresentados através de histogramas, um para cada cidade analisada, mostrando o número de caminhões rastreados, de hora em hora, por dia da semana em relação ao período de análise. Assim, o eixo x representa períodos de uma hora, das $00 \mathrm{~h}$ às $01 \mathrm{~h}$, da $01 \mathrm{~h}$ às $02 \mathrm{~h}$, e assim por diante; enquanto o eixo y representa o número de caminhões; e os dias da semana são representados por barras de cores diferentes em ordem cronológica, sendo a primeira referente à segunda-feira (06 de outubro de 2014) e a última à sexta-feira (10 de outubro de 2014).

Na Figura 2 apresenta-se, para o conjunto de dados da "Base A", o histograma do número de caminhões, cuja ordem de grandeza de caminhões rastreados durante uma semana em São Paulo é de 500 veículos. Observa-se que, embora haja variações na quantidade de caminhões circulando de terça à sexta, esta não é tão significativa, enquanto que o volume de caminhões que estão circulando na segunda-feira é inferior a $50 \%$ do volume observado nos demais dias da semana, sendo que essa diferença é mais acentuada nas primeiras horas do dia.

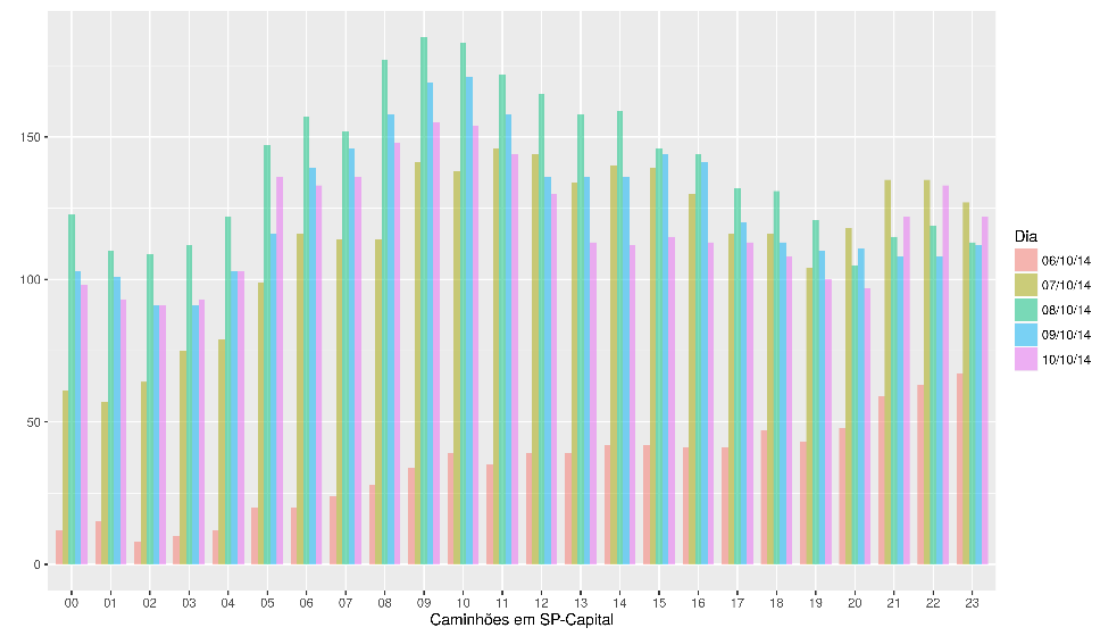

Figura 2: Número de caminhões (Base A) por dia da semana e hora do dia para São Paulo

Em relação a esta base de dados, apresenta-se o mesmo tipo de análise para a cidade de São Paulo (Figura 3) e algumas das principais capitais do país, a saber: Rio de Janeiro (Figura 4), Belo Horizonte (Figura 5), Curitiba (Figura 6), Recife (Figura 7) e Salvador (Figura 8). Nota-se que, exceto por Salvador, para todas as demais capitais é possível observar um padrão similar ao da Figura 2 ("Base A" - São Paulo) em especial em relação ao volume de caminhões circulando na segunda-feira ser bastante inferior ao observado em média nos demais dias da semana. 
Nota-se também que o padrão de distribuição do volume de caminhões rastreados ao longo do dia para a cidade de São Paulo é diferente na Base A (Figura 2) em comparação com a Base B (Figura 3). Cogita-se que isso provavelmente se deva às características das empresas as quais pertencem os veículos em cada base, embora não haja elementos nos dados obtidos que permitam verificar essa hipótese, particularmente no que tange à Base $\mathrm{B}$, que corresponde ao rastreamento de diversos tipos de veículos (caminhões, táxis, ônibus, automóveis) de um provedor de serviços de mapeamento.

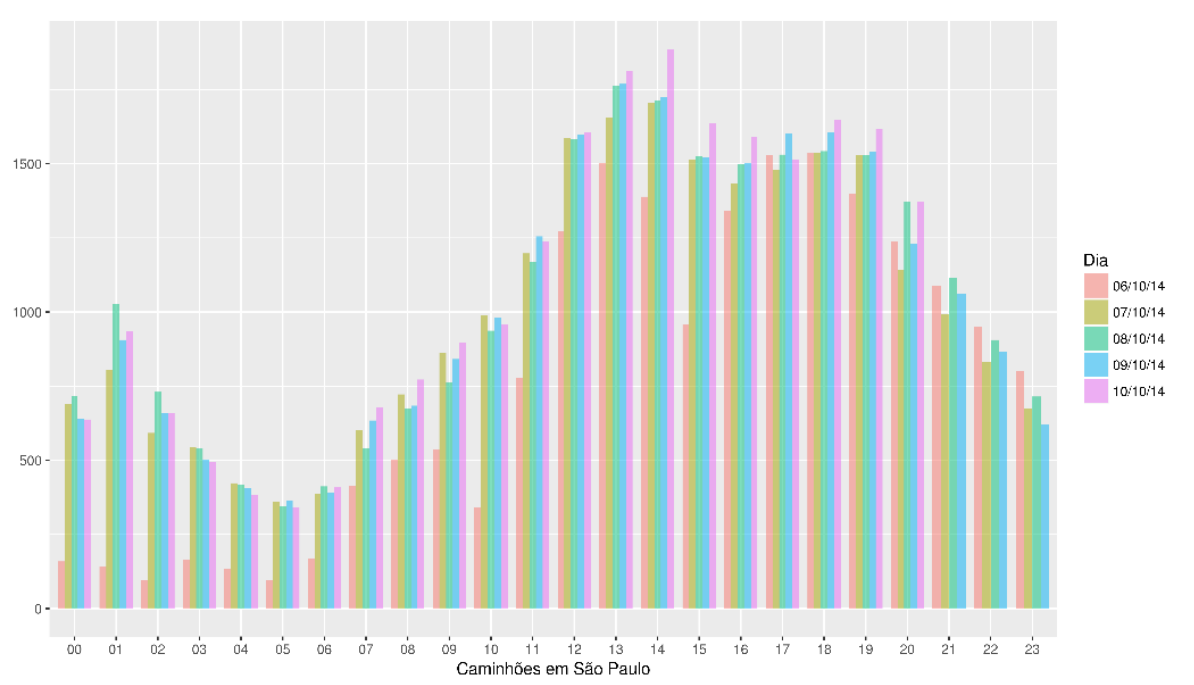

Figura 3: Número de caminhões (Base B) por dia da semana e hora do dia para São Paulo

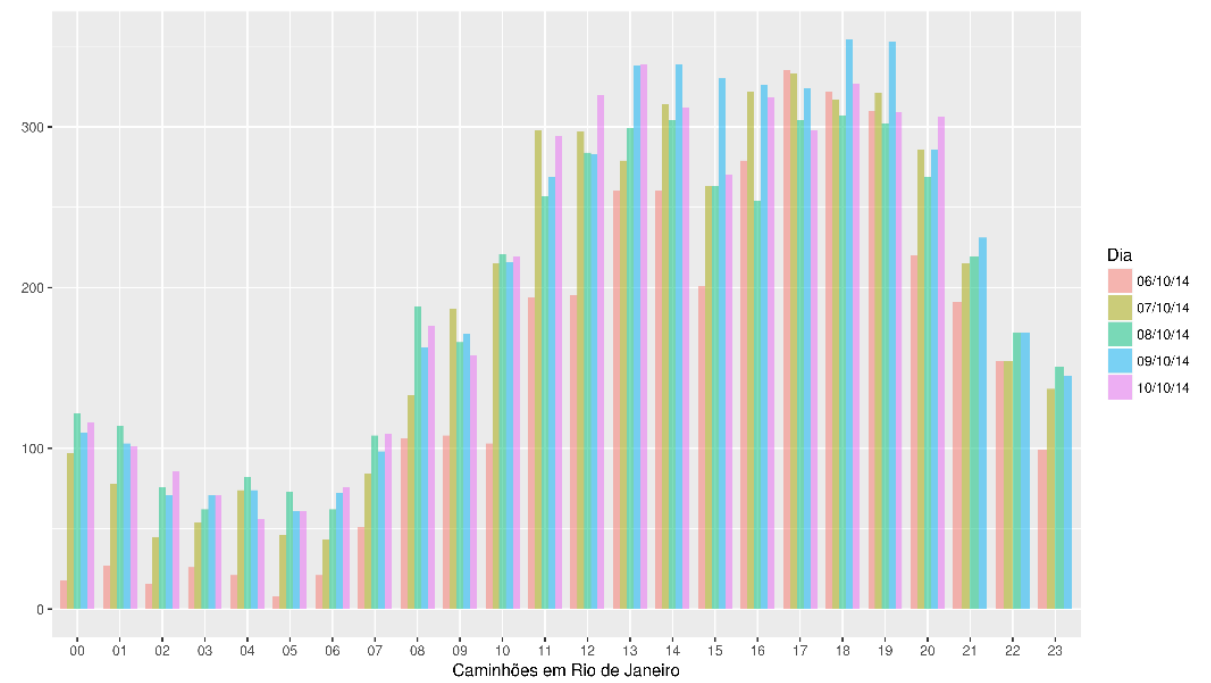

Figura 4: Número de caminhões (Base B) por dia da semana e hora do dia para Rio de Janeiro

Mais especificamente, observa-se que o pico do volume de caminhões se dá entre 08 e $10 \mathrm{~h}$ da manhã para a Base A, e o volume varia percentualmente menos do que na Base B ao longo do dia, ou seja, é mais distribuído; enquanto na Base $B$, o pico do volume de caminhões se encontra entre às $12 \mathrm{~h} e$ $14 \mathrm{~h}$, e o volume noturno é bastante inferior à média do período diurno. Com relação às outras capitais analisadas considerando-se a Base B, a distribuição do volume por hora do dia apresenta padrões diferentes: em alguns casos, como Curitiba, Recife e Salvador, há um pico das $12 \mathrm{~h}$ às $14 \mathrm{~h}$ e outro das $17 \mathrm{~h}$ às $19 \mathrm{~h}$, enquanto em Belo Horizonte, o volume se mantém no pico entre 10 e $19 \mathrm{~h}$. 


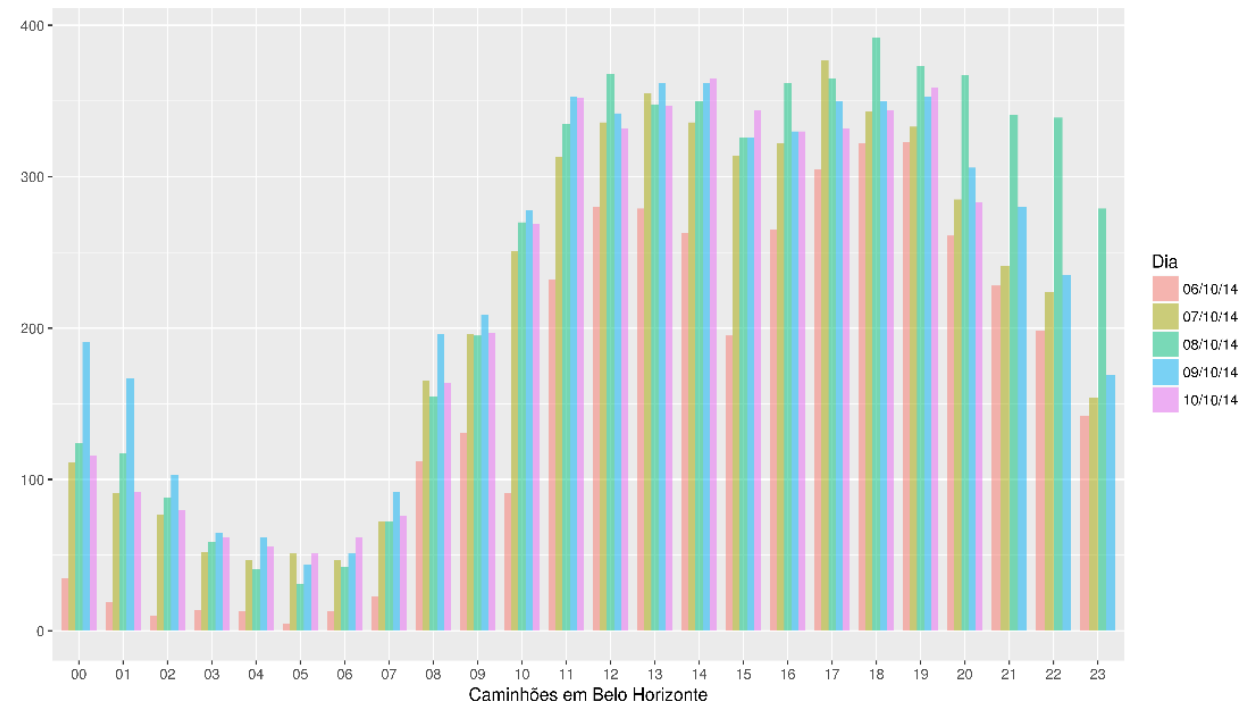

Figura 5: Número de caminhões (Base B) por dia da semana e hora do dia para Belo Horizonte

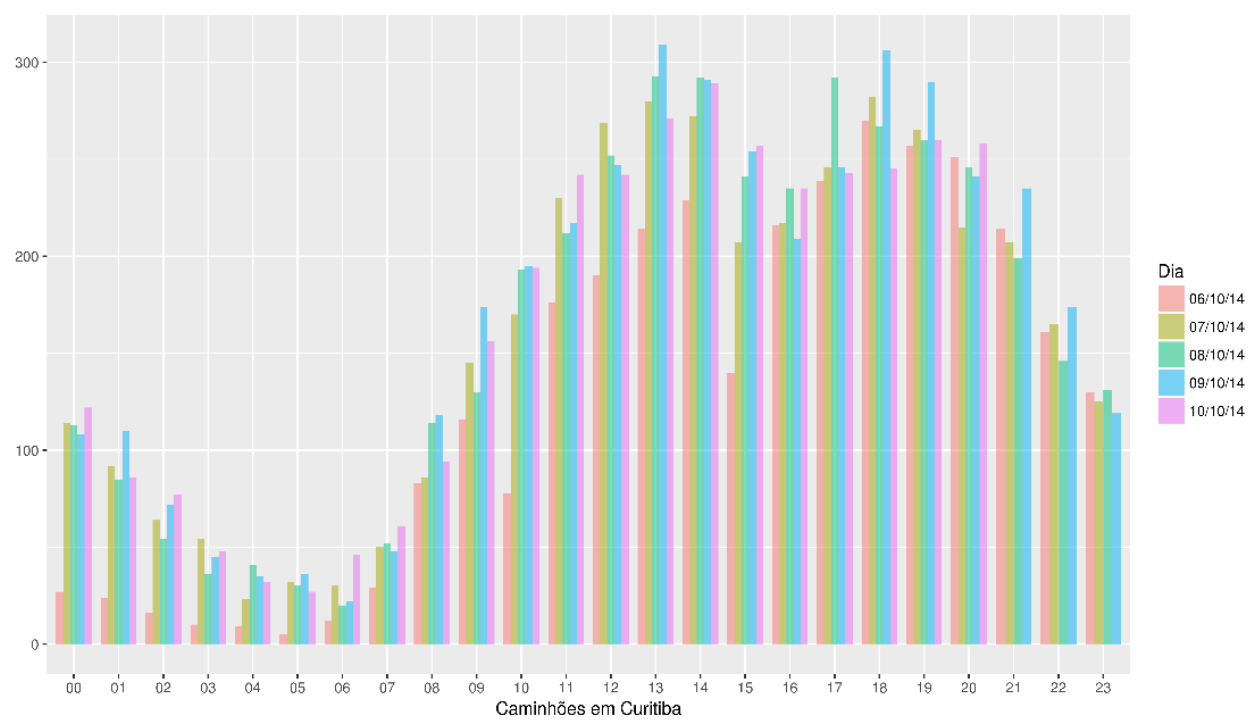

Figura 6: Número de caminhões (Base B) por dia da semana e hora do dia para Curitiba

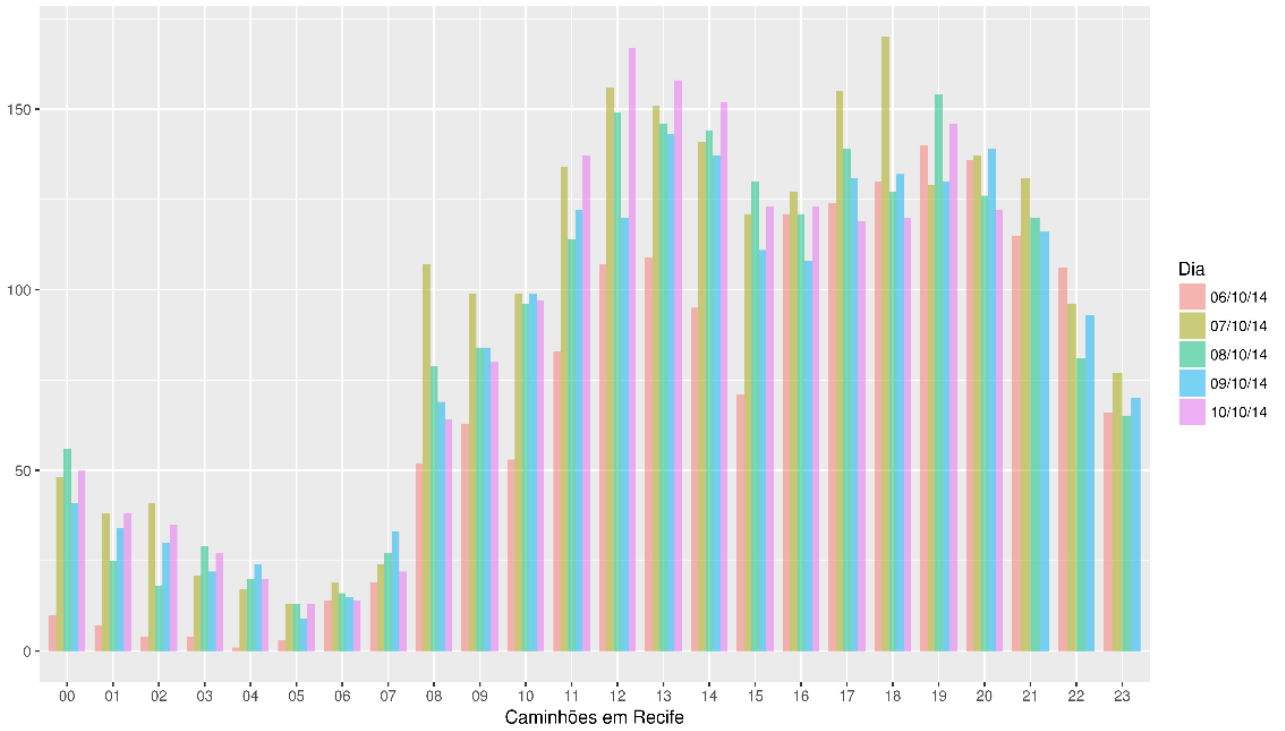

Figura 7: Número de caminhões (Base B) por dia da semana e hora do dia para Recife 
A Figura 9 apresenta o percentual do volume de caminhões que cada dia da semana (segunda à sexta) representa em relação ao volume total da semana analisada, para cada uma das capitais apresentadas anteriormente. Observa-se que o volume de segunda representa cerca $16 \%$ do total da semana, enquanto os demais dias representam cerca de $21 \%$ cada, ou seja, em média o volume de caminhões nas vias na segunda-feira é cerca de $25 \%$ menor que nos demais dias da semana, sendo essa diferença mais acentuada em Belo Horizonte, Curitiba e São Paulo.

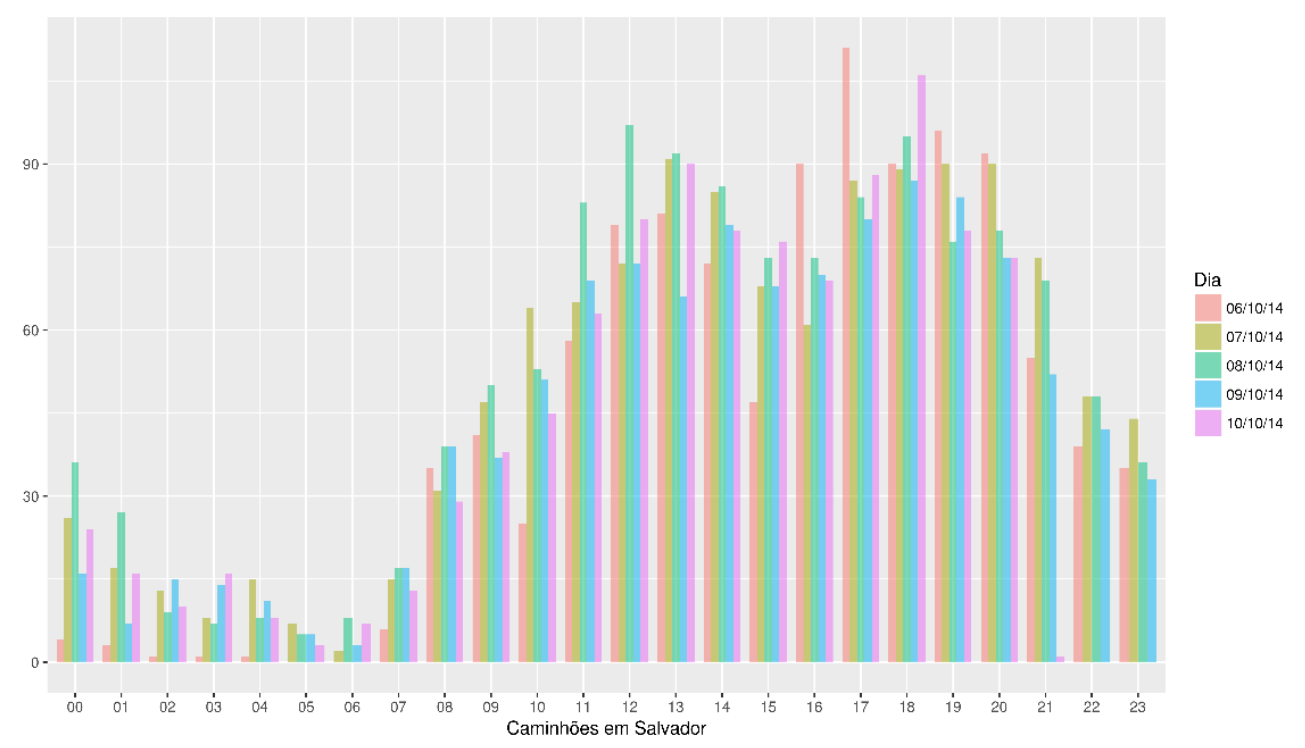

Figura 8: Número de caminhões (Base B) por dia da semana e hora do dia para Salvador

Na Figura 10 são apresentados mapas de calor com a densidade de registros de GPS dos caminhões na cidade de São Paulo, para cada dia da semana em análise, na ordem cronológica, de segunda à sexta. Tais figuras permitem analisar espacialmente se a distribuição da concentração desses veículos de carga também varia ao longo da semana. De maneira geral, nota-se que as densidades são muito semelhantes, com pequenas variações nas áreas de menor densidade (amarelo claro), sendo que na segunda essas áreas são menores, devido principalmente à quantidade menor de veículos circulando neste dia. Em outras palavras, observa-se que nessa escala os veículos de carga circulam nas mesmas regiões da cidade nos diferentes dias da semana, não havendo uma particularização nos diferentes dias.

Em relação ao sistema viário utilizado pelos veículos de carga, é possível notar que estes utilizam primordialmente as vias de fluxo mais rápido para se deslocar dentro da cidade de São Paulo, se concentrando nas Marginais (Tietê e Pinheiros) e nas vias arteriais. Adicionalmente, observa-se uma concentração também alta de caminhões circulando pelo Rodoanel e outras rodovias, o que é justificável dado que a grande parte dos centros de distribuição que consistem nos pontos de origem das viagens desses veículos está localizado em cidades vizinhas, em sua maioria, próximos às rodovias.

Este último ponto pode levantar dúvidas quanto à sustentabilidade da estratégia de sediar tais instalações logísticas mais longe das áreas de consumo que serão abastecidas a partir destas, no sentido de que isto acaba por gerar um deslocamento maior, ou seja, mais quilômetros percorridos, implicando também em mais emissões de gases de efeito estufa (GEE). Sabe-se, entretanto, que este fenômeno, conhecido como "dispersão logística" - do inglês "logistics sprawl", definido como "o movimento de instalações logísticas para fora da cidade" (Dablanc e Rakotonarivo, 2010) não é totalmente voluntário, ao contrário, está diretamente relacionado ao aumento do limite da área urbana, que por consequência, reflete no aumento do custo do metro quadrado de imóveis dessas áreas. Neste caso, caberia uma análise mais profunda no sentido de avaliar o impacto desse fenômeno, e possibilitar que o poder público tenha condições de considerar tais comportamentos ao tomar decisões em relação a políticas de mobilidade urbana para a cidade. 


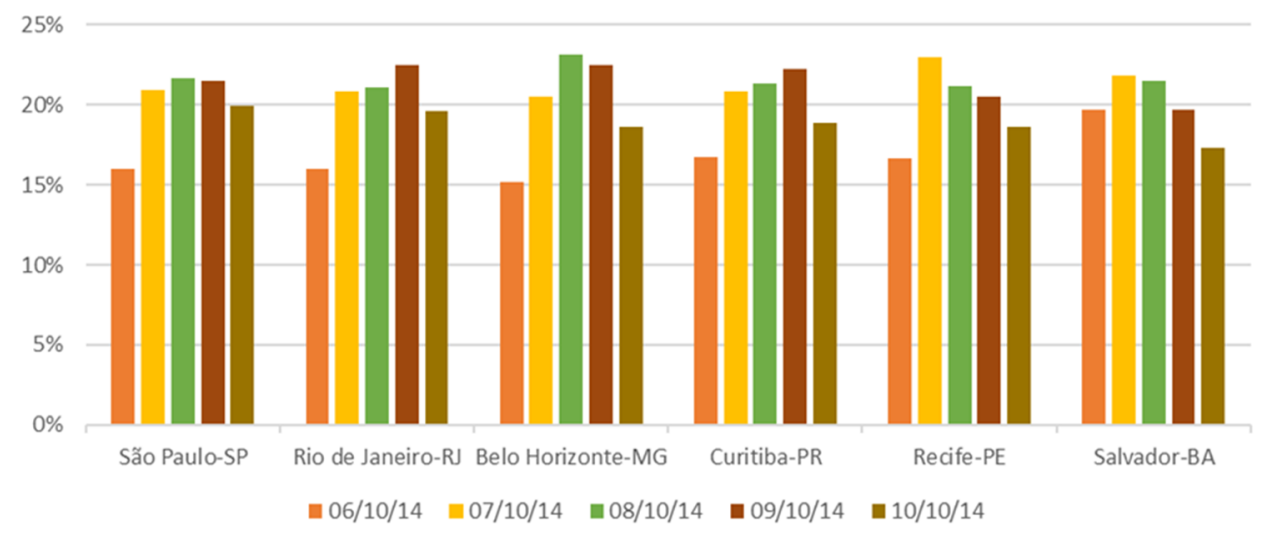

Figura 9. Número de caminhões (\%) por dia da semana (Base B)

\section{CONSIDERAÇÕES FINAIS}

As análises indicam fortes indícios de que o volume de caminhões circulando nas capitais às segundasfeiras seja razoavelmente inferior ao volume observado no restante dos dias (terças às sextas-feiras). Uma hipótese que pode justificar tal padrão observado, está relacionado ao fato de a maioria dos centros de distribuição não possuírem operação aos domingos, ou seja, ao retomar as operações às segundasfeiras é possível que os veículos demorem mais tempo para iniciar suas viagens com destino aos pontos de entrega, pois é necessário antes que haja todo o processamento dos pedidos, separação e carregamento da carga no veículo.

Tal aspecto observado nos resultados relativos à segunda-feira deve ser explorado mais profundamente, seja através de pesquisa junto aos transportadores, operadores logísticos e embarcadores de carga, ou ainda através de contagens de veículo classificadas para todos os dias da semana, de maneira a validar o padrão identificado. De qualquer forma, o fato de uma análise como esta levantar um possível padrão desconhecido do comportamento da logística urbana em grandes cidades, corrobora para confirmar a necessidade de mais estudos nessa área, os quais possam balizar novas políticas públicas, além de estratégias logísticas adotadas por empresas privadas em geral que, atualmente, têm como premissas hipóteses feitas sem uma base de conhecimento que as sustente de forma confiável.

Sabe-se que este campo de estudo que engloba a análise e visualização de dados e sua aplicação a dados de rastreamento é bastante novo, portanto ainda há muito a ser explorado. Ressalta-se que o custo de obtenção deste tipo de dados é bastante baixo, dado que muitas empresas já os têm por questões de gerenciamento de risco, corroborando para necessidade de se desenvolver formas eficazes de transformar tais dados em informações úteis e relevantes.

Tais informações poderiam ser aplicadas tanto para o setor privado no intuito de aumentar sua eficiência operacional e reduzir custos, como para o setor público no sentido de ter a possibilidade de fazer um diagnóstico da logística urbana e propor políticas públicas mais abrangentes e eficazes, podendo inclusive permitir entender e medir antecipadamente quais seriam os resultados de tais medidas de forma mais realista e confiável.

Dentre as possíveis análises sugere-se a avaliação da variação da distribuição da circulação de caminhões por período de tempo (mês, semana, dia, hora), inclusive espacialmente no sentido de identificar o viário utilizado por tais veículos. Outro aspecto bastante rico a ser explorado está relacionado a identificar os pontos de entrega destes veículos de carga, determinando assim os tempos de parada, tempos de deslocamento, e, portanto, também as respectivas velocidades. A partir dessas informações seria possível, por exemplo, identificar a demanda por vagas de carga e descarga por bairro/região da cidade, e aplicar tal informação para definir futuras políticas públicas. 


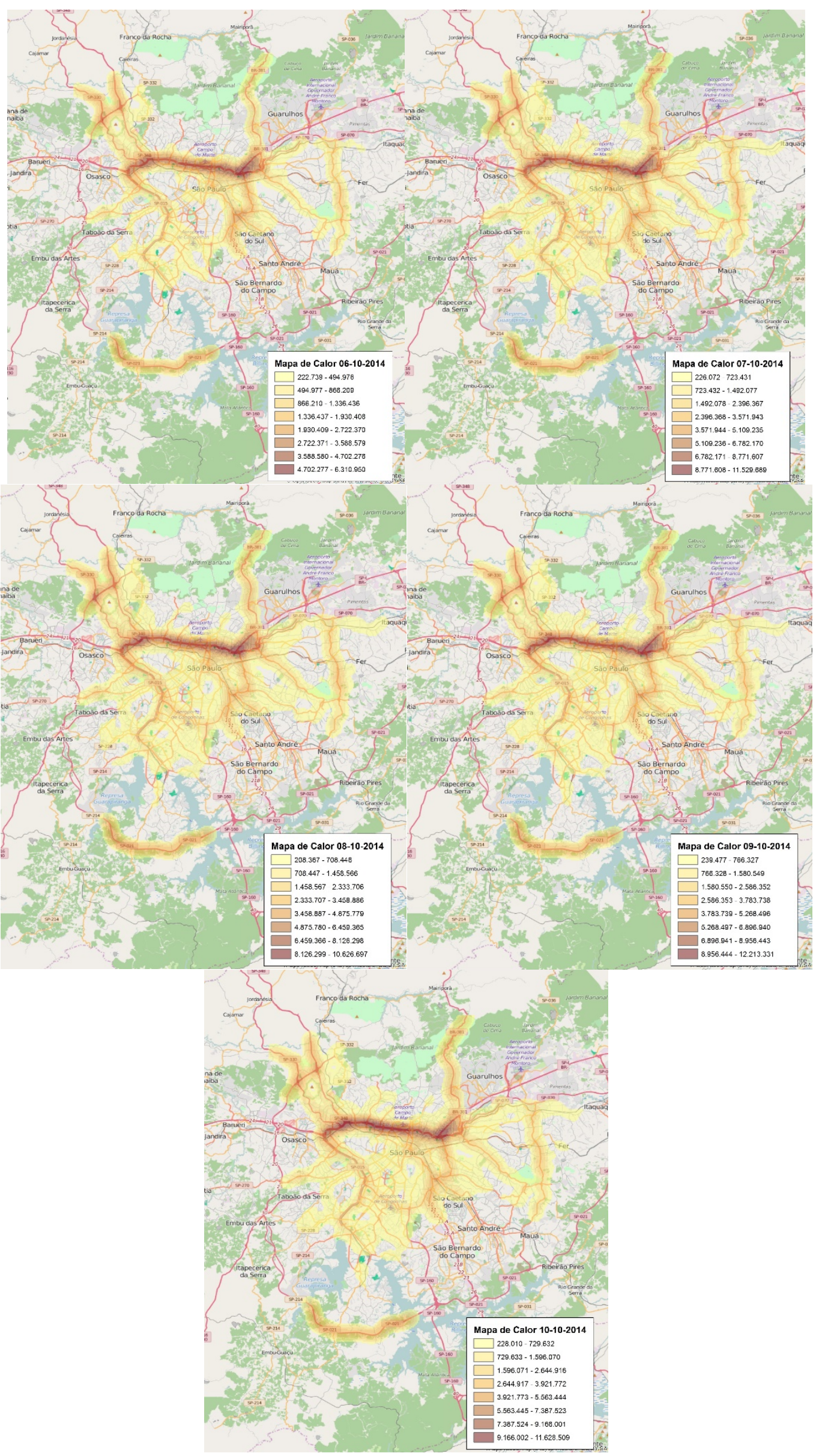

Figura 10: Densidade de registros dos caminhões (Base B) por dia da semana (2a a 6a feira) para São Paulo 


\section{REFERÊNCIAS}

Bontempo, A.P.; C.B. Cunha; D.A. Botter e H.T.Y. Yoshizaki (2014) Evaluating Restrictions on the Circulation of Freight Vehicles in Brazilian Cities. Procedia - Social and Behavioral Sciences, 125, p. 275-283. DOI:/10.1016/j.sbspro.2014.01.1473

CET-SP (2015) Pesquisa de Monitoração da Mobilidade no Sistema Viário Principal. Companhia de Engenharia de Tráfego de São Paulo, São Paulo, SP.

Chi, E.H. (2000) A taxonomy of visualization techniques using the data state reference model. Proceedings of the IEEE Symposium on Information Visualization InfoVis, IEEE Computer Society, Washington, DC, USA, p. 69-76. DOI:/10.1109/INFVIS.2000.885092

Dablanc, L. e D. Rakotonarivo (2010) The impacts of logistics sprawl: How does the location of parcel transport terminals affect the energy efficiency of goods' movements in Paris and what can we do about it?. Procedia-Social and Behavioral Sciences, 2(3), p. 6087-6096. DOI:/10.1016/j.sbspro.2010.04.021

Dorling, D.; A. Barford e M. Newman (2006) WORLDMAPPER: The world as you've never seen it before. IEEE transactions on visualization and computer graphics, 12(5), p. 757-764. DOI:/10.1109/TVCG.2006.202

Greaves, S. P. e M. A. Figliozzi (2008) Commercial Vehicle Tour Data Collection Using Passive GPS Technology: Issues and Potential Applications. Institute of Transport and Logistics Studies.

Joubert, J. W. e S. Meintjes (2015) Repeatability \& reproducibility: Implications of using GPS data for freight activity chains. Transportation Research Part B: Methodological, 76, p. 81-92. DOI:/10.1016/j.trb.2015.03.007

Kraak, M-J. e F. Ormeling (2011) Cartography: Visualization of Spatial Data, Guilford Press.

Lee, J-G e M. Kang (2015) Geospatial Big Data: Challenges and Opportunities.Big Data Research, v.2, p. 74-81. DOI: /10.1016/j.bdr.2015.01.003

Loidl, M.; G. Wallentin; R. Cyganski; A. Graser; J. Scholz e E. Haslauer (2016) GIS and Transport Modeling-Strengthening the Spatial Perspective. ISPRS International Journal of Geo-Information. DOI:/10.3390/ijgi5060084

Nanni, M. (2013) Mobility Data Mining. Cambridge: Cambridge University Press. DOI:/10.1017/CB09781139128926

Schumann, H. e C. Tominski (2011) Analytical, visual and interactive concepts for geo-visual analytics. Journal of Visual Languages and Computing 22, p. 257-267. DOI:/10.1016/j.jvlc.2011.03.002

Spaccapietra, S.; C. Parent e L. Spinsanti (2013) Trajectories and Their Representations. Cambridge University. DOI:/10.1017/CBO9781139128926.002

Tominski, C.; P. Schulze-Wollgast e H. Schumann (2005) 3D information visualization for time dependent data on maps. In Ninth International Conferenceon Information Visualisation, p. 175-181. IEEE. DOI:/10.1109/IV.2005.3 